Proceedings of the Third SIGdial Workshop on Discourse and Dialogue, Philadelphia, July 2002, pp. 162-173. Association for Computational Linguistics.

\title{
Training a Dialogue Act Tagger For Human-Human and Human-Computer Travel Dialogues
}

\author{
Rashmi Prasad and Marilyn Walker \\ AT\&T Labs - Research \\ 180 Park Avenue, Florham Park, NJ 07932, U.S.A. \\ rjprasad, walkereresearch.att.com
}

\begin{abstract}
While dialogue acts provide a useful schema for characterizing dialogue behaviors in human-computer and humanhuman dialogues, their utility is limited by the huge effort involved in handlabelling dialogues with a dialogue act labelling scheme. In this work, we examine whether it is possible to fully automate the tagging task with the goal of enabling rapid creation of corpora for evaluating spoken dialogue systems and comparing them to human-human dialogues. We report results for training and testing an automatic classifier to label the information provider's utterances in spoken human-computer and human-human dialogues with DATE (Dialogue Act Tagging for Evaluation) dialogue act tags. We train and test the DATE tagger on various combinations of the DARPA Communicator June-2000 and October-2001 human-computer corpora, and the CMU human-human corpus in the travel planning domain. Our results show that we can achieve high accuracies on the humancomputer data, and surprisingly, that the human-computer data improves accuracy on the human-human data, when only small amounts of human-human training data are available.
\end{abstract}

\section{Introduction}

Recent research on dialogue is based on the assumption that dialogue acts provide a useful way of characterizing dialogue behaviors in both humanhuman $(\mathrm{HH})$ and human-computer (HC) dialogue (Isard and Carletta, 1995; Shriberg et al., 2000; Di Eugenio et al., 1998; Cattoni et al., 2001). Previous research has used dialogue act tagging for tasks such as improving recognition performance (Shriberg et al., 2000), identifying important parts of a dialogue (Finke et al., 1998), evaluating and comparing spoken dialogue systems (Walker et al., 2001c; Cattoni et al., 2001; Hastie et al., 2002), as a constraint on nominal expression generation (Jordan, 2000), and for comparing $\mathrm{HH}$ to $\mathrm{HC}$ dialogues (Doran et al., 2001).

Our work builds directly on the previous application of the DATE (Dialogue Act Tagging for Evaluation) tagging scheme to the evaluation and comparison of DARPA Communicator dialogues. The hypothesis underlying the use of dialogue act tagging in spoken dialogue evaluation is that a system's dialogue behaviors have a strong effect on its usability. Because Communicator systems have unique dialogue strategies, and a unique way of representing and achieving particular communicative goals, DATE was developed to consistently label dialogue behaviors across systems so that the potential utility of dialogue act tagging could be explored. In previous work, Walker and Passonneau defined the DATE scheme, and labelled the system utterances in the June 2000 data collection of 663 dialogues from nine participating Communicator systems (Walker et al., 2001c; Walker et al., 2001a). They then derived dialogue act metrics from the DATE tags and showed that when these metrics were used in the PARADISE evaluation framework (Walker et al., 1997) that they improved models of user satisfaction by an absolute $5 \%$, and that the new metrics could be used to understand which system's dialogue strategies were most effective. 
A major part of evaluation effort using dialogue act tagging, however, is to actually label the dialogues with the dialogue act tags. In previous work (Walker et al., 2001c), the DATE labelling of the June-2000 corpus was done using a semi-automatic method that involved collection of a large number of utterance patterns from the different sites participating in the collection and subsequent hand labelling of these patterns. The $100 \%$ coverage and accuracy achieved by the pattern matcher that was implemented for labelling the system utterances was crucially at the cost of maintaining a large labelled pattern database. ${ }^{1}$ Furthermore, since the collected patterns were very specific and often exact duplicates of the system utterances in the dialogues, slight variations in the system utterances over time led to a reduction in coverage of the pattern matcher. For example, on the October-2001 collection, the tagger was able to label only $60 \%$ of the data. Accounting for the unmatched (and thus unlabeled) utterances turned out again to be a tedious process of manually augmenting the pattern database with new utterance patterns.

We examine whether it is possible to fully automate the tagging task with the goal being to enable rapid creation of new spoken dialogue corpora that can be submitted to evaluation procedures that rely on tagging schemes like DATE. Our hypothesis, arising from rough qualitative studies, is that within the same domain there are large similarities not only between different $\mathrm{HC}$ dialogues (across system as well as over a period of time) such as the Communicator dialogues, but also between $\mathrm{HC}$ dialogues and $\mathrm{HH}$ dialogues. Figure 1 shows a $\mathrm{HC}$ dialogue and Figure 2 shows a $\mathrm{HH}$ dialogue from the CMUcorpus ( $\mathrm{U}=$ user, $\mathrm{A}=$ human agent, $\mathrm{S}=$ computer system) in which the information providers' utterances for information gathering and information presentation across both dialogues are very similar. Thus, based on this and other similar observations, we decided to test the extent to which sufficiently general tagging rules can be learned from $\mathrm{HC}$ data to label other $\mathrm{HC}$ data and $\mathrm{HH}$ data in similar domains with high accuracy.

We focus on labelling only the information providers' side of the dialogues. The primary motivation for this is to be able to characterize system dialogue strategies for cross-site evaluation. System dialogue behaviors have a large impact on users' ex-

\footnotetext{
${ }^{1}$ It should be noted, however, that the task of labelling the utterances in the pattern database was still a considerable simplification over entire dialogues being labelled by human annotators, which is the usual practice in tagging tasks.
}

S: Leaving from Phoenix, And, what city are you flying to? U: SEOUL KOREA

S: Flying from Phoenix to Seoul, What date would you like to fly?

U: WEDNESDAY OCTOBER ELEVENTH

S: Leaving on the 11th of October, What time woodja like to depart?

U: ANY TIME

S: From Seoul. Are you returning to Phoenix?

U: YES

S: And what date didja wanna return?

U: SUNDAY OCTOBER FIFTEENTH

S: I may have misheard You saying the 5th of October.

Please be aware that the return date must be later than the

departure date. Please tell me what date you would like too return.

Figure 1: Excerpt of a HC Dialogue from the June2000 Corpus

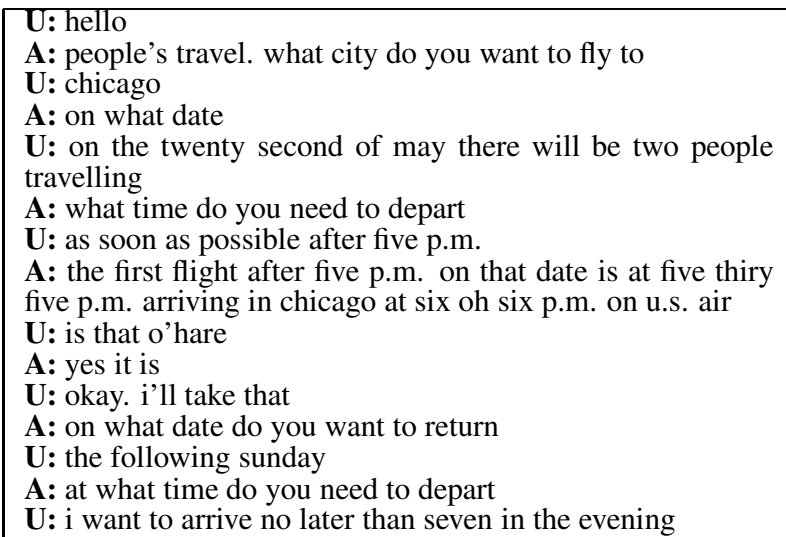

Figure 2: Excerpt of a HH Dialogue from the CMUCorpus

perience and on users' behavior. Furthermore, users in the $\mathrm{HC}$ dialogues rarely took initiative and their utterances showed very little variation (Doran et al., 2001). In addition, we believe that once the system side of the dialogues is labelled, it will be much easier to label the user side of the dialogues.

We report the results of applying a rule-induction method to train and test DATE taggers on various combinations of the DARPA Communicator June2000 and October-2001 HC corpora, and the CMU $\mathrm{HH}$ corpus in the travel planning domain. The accuracy of a DATE tagger trained and tested on the June-2000 corpus is $98.5 \%$. On the October-2001 corpus, this tagger achieves an accuracy of only $71.8 \%$, but adding 2000 utterances from the 2001 corpus to the training data improves accuracy on the rest of the 2001 corpus to $93.8 \%$. The accuracy of a tagger trained on the $\mathrm{HC}$ corpora and tested on the CMU-corpus is $36.7 \%$ (a significant improvement over the baseline of 28\%). A DATE tagger trained on 305 examples of the $\mathrm{HH}$ data achieves 
an accuracy of $48.75 \%$, but the addition of the HC training data improves accuracy to $55.5 \%$ (majority class baseline $=28 \%$ ). This pair of results demonstrates quantitatively that the $\mathrm{HC}$ data can be used to improve performance of a tagger for $\mathrm{HH}$ data. However, a larger training corpus of $\mathrm{HH}$ data improves performance to $76.6 \%$ accuracy, as estimated by 20 fold cross-validation on the CMU-corpus.

Section 2 describes the corpora, the DATE dialogue act tagging scheme, methods for tagging the corpora for the experiments, and the features used to train a DATE dialogue act tagger for DATE labelling of the corpora. Section 3 presents our results. We postpone discussion and comparison with related work till Section 4 .

\section{Corpus, Data, Methods}

Our experiments apply the rule learning program RIPPER (Cohen, 1996) to train a DATE dialogue act tagger for the utterances of the information provider in $\mathrm{HC}$ and $\mathrm{HH}$ travel planning dialogues. Like other automatic classifiers, RIPPER takes as input the names of a set of classes to be learned, the names and ranges of values of a fixed set of features, and training data specifying the class and feature values for each example in a training set. Its output is a classification model for predicting the class of future examples. In RIPPER, the classification model is learned using greedy search guided by an information gain metric, and is expressed as an ordered set of if-then rules. Although any of several automatic classifiers could be used to train an automatic DATE tagger, RIPPER supports textual features, which are important for this problem, and outputs if-then rules that are easy to understand and which make clear which features are useful to the DATE tagger when classifying utterances.

To apply RIPPER, the utterances in the corpus must be encoded in terms of a set of classes (the output classification) and a set of input features that are used as predictors for the classes. Below we describe the corpora, the classes derived from the DATE tagging scheme, the methods used for tagging the corpora using the DATE scheme, and the features that are extracted from the dialogue in which each utterance occurs.

\subsection{Travel Planning Corpora}

Our experiments utilize both $\mathrm{HC}$ and $\mathrm{HH}$ dialogues in the travel planning domain. The DARPA Communicator HC dialogue corpus consists of the June2000 corpus and the October-2001 corpus. The
June-2000 corpus contains 663 experimental dialogues collected during a three week period in June of 2000 for conversations between human users and 9 different Communicator travel planning systems. The October-2001 corpus contains 1252 experimental dialogues collected between April and October of 2001 for conversations between human users and 8 different COMMUNICATOR travel planning systems. The dialogues were quite complex, ranging between simple one way trips requiring no ground arrangements to multileg trips to international or domestic destinations that required car and hotel arrangements. The dialogues typically lasted between 2 and 10 minutes. There was a great deal of variation in the dialogue strategies implemented by the different systems, both between the sites during each collection as well as within a single site across the different collections, from 2000 to 2001 . There were a total of 22930 system utterances in the June- 2000 corpus and a total of 69766 utterances in the October2001 corpus. Each dialogue interaction was logged by each system using a shared logfile standard. We were primarily interested in three logged features: (1) the text of each system utterance; (2) what the recognizer understood for each user utterance; and (3) the transcription that each site provided for what the user actually said. We describe below in Section 2.4 how we used these three logfile features to derive the features used to train the DATE tagger.

The HH dialogue corpus consists of the CMUcorpus (Eskenazi et al., 1999). Dialogues in the travel planning domain were collected by the Communicator group at Carnegie Mellon University (CMU), who arranged with the onsite travel agency People's Travel to record calls from a number of volunteer subjects who called the human travel agent to plan intended trips. These calls were then transcribed and the recordings and the transcriptions were made available to members of the Communicator community. Labellers at our site subsequently segmented the travel agent side of the conversation into utterances where each utterance realized a single dialogue act. We used this utterance level segmentation to define the unit for tagging in the experiments described below. The CMU-corpus consists of 38 dialogues with a total of 1062 travel agent utterances.

\subsection{Class Assignment}

The classes used to train the DATE tagger are derived directly from the DATE tagging scheme (Walker et al., 2001c). DATE classifies each utterance along three cross-cutting orthogonal dimen- 
sions of utterance classification: (1) a SPEECH ACT dimension; (2) a CONVERSATIONAL-DOMAIN dimension; and (3) a TASK-SUBTASK dimension. The SPEECH ACT and CONVERSATIONAL-DOMAIN dimensions should be general across domains, while the TASK-SUBTASK dimension involves a task model that is not only domain specific, but could vary from system to system because some systems might make finer-grained subtask distinctions.

The SPEECH ACT dimension captures distinctions between distinct communicative goals such as requesting information (REQUEST-INFO), presenting information (PRESENT-INFO) and making offers (OFFER) to act on behalf of the caller. The types of speech acts are specified and illustrated in Figure 3.

\begin{tabular}{|ll|}
\hline Speech-Act & Example \\
\hline \hline REQUEST-INFO & And, what city are you flying to? \\
\hline PRESENT-INFO & $\begin{array}{l}\text { The airfare for this trip is } 390 \text { dol- } \\
\text { lars. }\end{array}$ \\
\hline OFFER & $\begin{array}{l}\text { Would you like me to hold this op- } \\
\text { tion? }\end{array}$ \\
\hline ACKNOWLEDGMENT & I will book this leg. \\
\hline BACKCHANNEL & Okay. \\
\hline STATUS-REPORT & $\begin{array}{l}\text { Accessing the database; this might } \\
\text { take a few seconds. }\end{array}$ \\
\hline $\begin{array}{l}\text { EXPLICIT- } \\
\text { CONFIRM }\end{array}$ & $\begin{array}{l}\text { You will depart on September } 1 \text { st. Is } \\
\text { that correct } ?\end{array}$ \\
\hline $\begin{array}{l}\text { IMPLICIT- } \\
\text { CONFIRM }\end{array}$ & Leaving from Dallas. \\
\hline INSTRUCTION & Try saying a short sentence. \\
\hline APOLOGY & Sorry, Ididn't understand that. \\
\hline $\begin{array}{l}\text { OPENING- } \\
\text { CLOSING }\end{array}$ & $\begin{array}{l}\text { Hello. Welcome to the C MUnicator. } \\
\text { mu Com- }\end{array}$ \\
\hline
\end{tabular}

Figure 3: Example Speech Acts in DATE

The CONVERSATIONAL-DOMAIN dimension distinguishes between talk devoted to the task of booking airline reservations ("about-task") versus talk devoted to maintaining the verbal channel of communication ("about-communication") (Allen and Core, 1997). DATE adds a third domain called "about-situation-frame", to distinguish utterances that provide information about the interactional context, e.g. Try saying a short sentence, or I know about 500 international destinations.

The TASK-SUBTASK dimension focusses on specifying which subtask of the travel reservation task the utterance contributes to. Some examples are given in Figure 4. This dimension distinguishes among 28 subtasks, some of which can also be grouped at a level below the top level task. The TOP-LEVEL-TRIP task describes the task which contains as its subtasks the ORIGIN, DESTINATION, DATE, TIME，AIRLINE，TRIP-TYPE，RETRIEVAL and ITINERARY tasks. The GROUND task includes both the HOTEL and CAR subtasks. The HOTEL task includes both the HOTEL-NAME and HOTELLOCATION subtasks.

Some utterances, especially about-situationframe utterances such as instructions and apologies are not specific to any task. For example, apologies made by the system about a misunderstanding can be made within any subtask. We give these utterances a "meta" value in the task dimension.

\begin{tabular}{|c|c|}
\hline Task & Example \\
\hline $\begin{array}{l}\text { TOP-LEVEL- } \\
\text { TRIP }\end{array}$ & What are your travel plans? \\
\hline ORIGIN & And, what city are you leaving from? \\
\hline DESTINATION & And, where are you flying to? \\
\hline DATE & What day would you like to leave? \\
\hline TIME & Departing at what time?. \\
\hline AIRLINE & Did you have an airline preference? \\
\hline RETRIEVAL & $\begin{array}{l}\text { Accessing the database; this might take a } \\
\text { few seconds. }\end{array}$ \\
\hline ITINERARY & The airfare for this trip is 390 dollars. \\
\hline GROUND & $\begin{array}{l}\text { Did you need to make any ground arrange- } \\
\text { ments?. }\end{array}$ \\
\hline HOTEL & Did you need a hotel?. \\
\hline $\begin{array}{l}\text { HOTEL- } \\
\text { NAME }\end{array}$ & Do you have a preferred hotel chain?. \\
\hline $\begin{array}{l}\text { HOTEL- } \\
\text { LOCATION }\end{array}$ & $\begin{array}{l}\text { Would you like a hotel near downtown or } \\
\text { near the airport?. }\end{array}$ \\
\hline CAR & Do you need a car in San Jose? \\
\hline CAR-TYPE & What kind of car did you want? \\
\hline CAR-RENTAL & Do you have a preferred rental agency? \\
\hline
\end{tabular}

Figure 4: Example Subtasks in DATE

It is possible to achieve very specific labelling of system utterances by applying all three dimensions simultaneously. For example, one set of output classes for the DATE tagger consists of the combination of all three classes so that an utterance such as I found three flights that match your request is classified as ABOUT-TASK:PRESENTINFO:FLIGHT. ${ }^{2}$ However, the DATE scheme also makes it possible to train and test a DATE tagger for just the SPEECH-ACT dimension or just the TASK dimension. Figure 5 shows utterances from a June-2000 dialogue fragment that are classified along each of the three DATE dimensions.

Tagging utterances along the SPEECH ACT dimension provides the most general tagging. This level of categorization is task-independent and possibly situation independent, ie. from $\mathrm{HC}$ to $\mathrm{HH}$ dialogues. One set of experiments simply tests performance of a DATE tagger for the speech-act dimension on the $\mathrm{HC}$ dialogue data. In addition, we also train a DATE tagger on the $\mathrm{HC}$ dialogues using only the speech

${ }^{2}$ DATE labels that are specified for all the three dimensions have the dimension values given in three fields separated by ":". The first field contains the value for the Conversational-Domain Dimension, the second for the Speech-Act Dimension, and the third for the Task-Subtask Dimension. 
act dimension for the purpose of applying it to a test set of the CMU-corpus of $\mathrm{HH}$ dialogues. ${ }^{3}$

\subsection{Preparation of Training and Test Data via DATE Tagging}

The DATE labelling of the June-2000 data was done with a semi-automatic tagger: an utterance or utterance sequence is identified and labelled automatically by reference to a database of utterance patterns hand-labelled with DATE tags. The collection and DATE labelling of the utterance patterns was done in cooperation with site developers. As discussed above, these patterns for the 2000 data set were often quite specific, and often involved whole utterances. However, since the systems use template based generation and have only a limited number of ways of saying the same content, relatively few utterance patterns needed to be hand-labelled when compared to the actual number of utterances occurring in the corpus. Further abstraction on the patterns was done with a named-entity labeller which replaces specific tokens of city names, airports, hotels, airlines, dates, times, cars, and car rental companies with their generic type labels. For example, what time do you want to leave $<A I R P O R T>$ on $<D A T E-T I M E>$ ? is the typed utterance for what time do you want to leave Newark International on Monday?. For the 2000 tagging, the number of utterances in the pattern database was 1700 whereas the total number of utterances in the 663 dialogues was 22930 . The named-entity labeller was also applied to the system utterances in the corpus. We collected vocabulary lists from all the sites for the named-entity labelling task. In most cases, systems had preclassified the individual tokens into generic types.

The tagger implements a simple pattern matching algorithm to do the dialogue act labelling: for each utterance pattern in the pattern database, the tagger attempts to find a match in the dialogues; if the match succeeds, the DATE label of that pattern is assigned to the matching utterance in the dialogue. The matching ignores punctuation since systems vary in the way they record punctuation. ${ }^{4}$

Certain utterances have different communicative functions depending on the context in which they

\footnotetext{
${ }^{3}$ Tagging utterances along the TASK dimension may provide a rough notion of discourse segmentation in that utterances about the same task may be grouped together. Due to lack of space, however, we do not present results for task tagging.

${ }^{4}$ Ignoring punctuation does not, however, create an utterance segmentation problem for the tagger. We assume that the utterances in the pattern database provide the reference points for utterance boundaries.
}

occur. For example, phrases like leaving in the $<D A T E-T I M E>$ are implicit confirmations when they constitute an utterance on their own, but are part of the flight information presentation when they occur embedded in utterances such as I have one flight leaving in the $<D A T E-T I M E>$. To prevent incorrect labelling for such ambiguous cases, the pattern database is sorted so that sub-patterns are listed later than the patterns within which they are embedded, and the pattern matcher is forced to match patterns in their order of occurrence in the database.

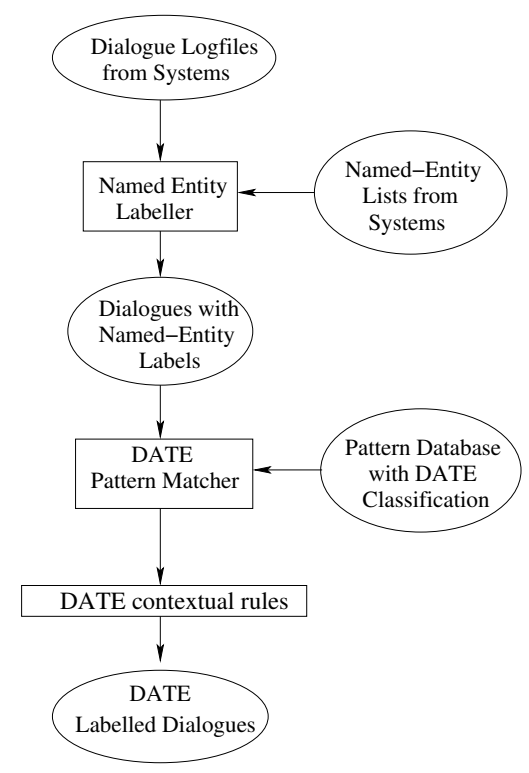

Figure 6: The DATE Dialogue Act Tagger

While this tagger achieved $100 \%$ accuracy for the 2000 data by using many specific patterns, when applied to the 2001 corpus it was able to label only $60 \%$ of the data. On examination of the unlabelled utterances, we found that many systems had augmented their inventory of named-entity items as well as system utterances from the 2000 to the 2001 data collection. As a result, there were many new patterns unaccounted for in the existing named-entity lists as well as in the pattern database. In an attempt to cover the remaining $40 \%$ of the data, we therefore augmented the named-entity lists by obtaining a new set of preclassified vocabulary items from the sites, and added 800 hand-labelled patterns to the pattern database. For the labelling of any additional unaccounted-for patterns, we implemented a contextual rule-based postprocessor that looks at the surrounding dialogue acts of an unmatched utterance within a turn and attempts to label it. The contextual rules are intended to capture 
rigid system dialogue behaviors that are reflected in the DATE sequences within a turn. ${ }^{5}$ For example, one very frequently occurring DATE sequence within system turns is about_task:present_info:flight, about_task:present_info:price, about_task:offer:flight. The rule using this contextual information can be informally stated as follows: if in a turn, the first two utterances are labelled as about_task:present_info:flight and about_task:present_info:price, and the third utterance is unlabelled, assign the third utterance the label about_task:offer:flight. Not all turn-internal DATE sequences are used as contextual rules, however, because many of them are highly ambiguous. For example, about_communicaton:apology:meta_slu_reject can be followed by a system instruction as well as any kind of request for information (typically) repeated from the previous system utterance. Figure 6 shows the current DATE tagging system, augmented with the DATE rule-based postprocessor.

With the 2000 tagger augmented with the additional named-entity items, utterance patterns, and the postprocessor, we were able to label $98.4 \%$ of the (69766) utterances in the 2001 corpus.

We conducted a hand evaluation of 10 dialogues which we selected randomly from each system. The evaluation of the total 80 dialogues shows that we achieved $96 \%$ accuracy on the 2001 tagging.

In order to label the HH corpus of 1062 utterances, we started with 10 dialogues (305 utterances) labelled with the CSTAR dialogue act tagging scheme (Finke et al., 1998; Doran et al., 2001). We automatically converted the labels to DATE, and then hand-corrected them. We labelled the rest of the HH data by training a DATE tagger, applying it to the remainder of the corpus, and hand-correcting the results.

\subsection{Feature Extraction}

The corpus is used to construct the machine learning features as follows. In RIPPER, feature values are continuous (numeric), set-valued (textual), or symbolic. We encoded each utterance in terms of a set of 19 features that were either derived from the logfiles, derived from human transcription of the user utterances, or represent aspects of the dialogue context in which each utterance occurs.

The complete feature set used by the machine learner is described in Figure 7. The features fall into three categories: (1) target utterance features ; (2) context features ; and (3) whole dialogue features.

\footnotetext{
${ }^{5}$ The logfile standard distinguishes system and user turns within the dialogues.
}

- target utterance features: utt-string, contains-wordFLIGHT-or-AIRLINE, contains-word-HOTEL-orROOM, contains-word-RENTAL-or-CAR, containsword-CITY-or-AIRPORT, contains-word-DATE-TIME, pattern-length.

- context features: left-sys-utt-string, right-sys-uttstring, da-num, position-in-turn, left-dacontext1, left-da-context2, usr-orig-string, usr-typed-string, rec-orig-string, rec-typed-string usr-rec-string-identity.

- whole dialogue features: system-name, turn-number.

Figure 7: Features used by the Machine Learner

The target utterance features include the target utterance string for which the dialogue act is to be predicted (utt-string), and a set of features derived from the named-entity labelling about what semantic types are instantiated in the target string. For example the feature contains-word-FLIGHTor-AIRLINE is represented by a boolean variable specifying whether the utterance string contains the words FLIGHT or AIRLINE. Similar features are contains-word-HOTEL-or-ROOM, containsword-RENTAL-or-CAR, contains-word-CITY-orAIRPORT, and contains-word-DATE-TIME. The pattern-length feature encodes the character length of the target utterance. The motivation for these features is to represent basic aspects of the target utterance, e.g. its length, and the lexical items and semantic types that appear in the utterance.

The context features encode simple aspects about the context in which the target utterance occurs. Two of these represent the system utterance strings to the left and right of the target utterance (left-sys-utt-string and right-sys-utt-string). The left-da-context 1 and left-da-context 2 features represent the left unigram and bigram dialogue act context of the target utterance; this goes beyond the target turn to only the last dialogue act in the previous system turn. The da-num feature encodes the number of dialogue acts in the target turn and the position-in-turn feature encodes the position of the target utterance in its turn. In addition, the user's previous utterance is represented as part of the context, both in terms of automatically extractable features like what the automatic speech recognizer thought the user said (rec-orig-string), and a version of this on which the named-entity labeller has been run (rec-typed-string), as well as in terms of human generated transcriptions of the user's utterance. Features based on the transcriptions include the original human transcription (usr-orig-string) and the transcription after named-entity tagging (usrtyped-string). The usr-rec-string-identity feature is a 


\begin{tabular}{|l|l|l|l|ll|}
\hline Training Data & Test Data & Dim & Maj. Cl. Baseline & Acc. & (SE) \\
\hline \hline JUNE-2000 & 4fold Xval JUNE-2000 & All & $6.45 \%$ & $98.5 \%$ & $0.11 \%$ \\
\hline JUNE-2000 & OCTOBER-2001 & All & $9.52 \%$ & $71.82 \%$ & $0.17 \%$ \\
\hline $\begin{array}{l}\text { JUNE-2000 \& 2000 ex- } \\
\text { amples of October-2001 }\end{array}$ & October-2001 w/out 2000 & All & $10.18 \%$ & $93.82 \%$ & $0.09 \%$ \\
\hline
\end{tabular}

Table 1: Results for Identifying Three-Way DATE Tags in the October-2001 Communicator Corpus, (Dim $=$ Dimension of Date used for output classification $($ Maj. Cl. $=$ Majority Class, Acc $=$ Accuracy, $\mathrm{SE}=$ Standard Error)

boolean feature based on comparing the user's transcribed utterance with the recognizer's hypothesis of what the user said, using simple string-identity. Some applications of DATE tagging would not use features derived from human generated transcriptions so the experiments below report accuracy figures for DATE taggers which ignore these features. The motivation for the context features is to represent aspects of the context in which the utterance occurs in terms of a window of surrounding lexical items and dialogue acts.

The whole dialogue features are the name of the site whose system generated the dialogue (systemname), and the turn number of the target utterance within the whole dialogue (turn-number). For $\mathrm{HH}$ dialogues the system-name has the value "human". The motivation for including the system-name feature is to see whether there are any aspects of the dialogue act realizations that are specific to particular systems. The motivation for the turn-number feature is that particular types of dialogue acts are more likely to occur in particular phases of the dialogue.

\section{Results}

Given the corpora and features described above, we constructed a set of training and test files for use with the RIPPER engine. Each spoken dialogue utterance by the system or by the human travel agent in the corpora are represented in terms of the features and class values described above. One of the primary goals in these experiments is to test the ability of the trained DATE tagger to learn and apply general rules for dialogue act tagging. In the HC data, we examine how a DATE tagger trained on the June-2000 corpus performs on the October-2001 corpus, with and without 2000 labelled examples of October-2001 training data. For the $\mathrm{HH}$ data, we examine how a DATE tagger trained on the two HC corpora (June-2000 and October-2001) performs on the CMU-corpus, with and without 305 utterances of $\mathrm{HH}$ labelled training data. We first report accuracy results for a DATE tagger trained and tested on the
HC June-2000 and October-2001 corpora and then report results for the HH CMU-corpus.

Human-Computer Results: Table 1 shows that the reported accuracies for the $\mathrm{HC}$ experiments are signifcantly better than the baseline in each case and the differences between the rows are also statistically significant. The first row shows that the accuracy of a DATE tagger trained and tested using four-fold cross-validation on the June-2000 data is $98.5 \%$ with a standard error of only $0.11 \%$. This indicates that after training on $75 \%$ of the data, there are few unexpected utterances in the remaining $25 \%$. However, the second row shows that a DATE tagger trained on the 9 systems represented in the June-2000 corpus and tested on the (subset) 8 systems represented in the October-2001 corpus only achieves $71.82 \%$ accuracy. This roughly matches our earlier finding in Section 2.3 that during the interval from June-2000 to April-2001 when the 2001 data collection began, many changes had been made to the Communicator systems and that the learned rules from the June-2000 data were not able to generalize as well to the October-2001 corpus.. The third row shows that the overall variation in the data is still low: when 2000 labelled examples of the October-2001 data are added to the June-2000 data for training, the accuracy increases to $93.82 \%$. This suggests that adding a small amount of new labelled training data for successive versions of a system would support high accuracy DATE tagging for the new version of the system.

Some of the rules that RIPPER learned from the $\mathrm{HC}$ corpora for predicting the DATE tag for utterances requesting information about the origin city, e.g. What city are you departing from?, and requesting information about the destination city, e.g. Where are you traveling to?, are shown in Figure 8. The figure shows that all of the rules for both about_task:request_info:orig_city and small about_task:request_info:dest_city utilize the utterance string feature. This suggests that single words in utterances can be regarded as reliable indicators 


\begin{tabular}{|l|l|l|l|ll|}
\hline Training Data & Test Data & Dim & Maj. Cl. Baseline & Acc. & (SE) \\
\hline \hline JUNE-2000 & 4fold Xval JUNE-2000 & SPA & $31.28 \%$ & $99.1 \%$ & $.09 \%$ \\
\hline JUNE-2000 & OCTOBER-2001 & SPA & $31.28 \%$ & $82.57 \%$ & $0.14 \%$ \\
\hline $\begin{array}{l}\text { JUNE-2000 \& 2000 ex- } \\
\text { amples of October-2001 }\end{array}$ & October-2001 w/out 2000 & SPA & $30.88 \%$ & $95.68 \%$ & $0.08 \%$ \\
\hline
\end{tabular}

Table 2: Results for Identifying Speech-Act DATE tags in the October-2001 Communicator Corpus, (Dim $=$ Dimension of Date used for output classification $(\mathrm{SPA}=$ Speech Act, Maj. Cl. = Majority Class, Acc $=$ Accuracy, SE $=$ Standard Error)

of DATE tags. More interestingly, the words utilized are intuitively plausible for the travel planning domain. For example, the learned question words such as which, where and would are significant for utterances that have request_info as their SPEECHACT dimension. The words city, airport, from, destination and departing are significant predictors of utterances that have orig_city and dest_city as their task dimension.

if utt-string contains city $\wedge$ utt-string contains from $\wedge$ pattern-length $\leq 38$

or if utt-string contains airport $\wedge$ pattern-length $\leq 38$

or if utt-string contains city $\wedge$ pattern-length $\leq 1 \overline{7} \wedge$ patternlength $>15$

or if utt-string contains from $\wedge$ pattern-length $\leq 66 \wedge$ uttstring contains Where

or if utt-string contains city $\wedge$ utt-string contains say

or if utt-string contains DEPARTING

or if utt-string contains which $\wedge$ utt-string contains From

or if utt-string contains city $\wedge$ system-name $=$ IBM $\wedge$ uttstring contains departure

or if utt-string contains fly $\wedge$ utt-string contains which $\wedge$ left-sys-utt-string contains city

or if utt-string contains fly $\wedge$ utt-string contains $\mathrm{O}$

then about_task:request_info:orig_city

if utt-string contains where $\wedge$ utt-string contains must

or if utt-string contains city $\wedge$ pattern-length $\leq 35$

or if utt-string contains Where

or if utt-string contains destination

or if utt-string contains DESTINATION

or if utt-string contains which $\wedge$ utt-string contains city

or if utt-string contains where

or if utt-string contains WOULD

then about_task:request_info:dest_city

Figure 8: Rules for DATE tags about_task:request_info:orig_city about_task:request_info:dest_city for Training on the June-2000 Corpus and 2000 Examples of October-2001 Corpus.

Human-Computer Speech-Act Results: Because the DATE scheme describes utterances in terms of SPEECH-ACT, CONVERSATIONALDOMAIN and TASK dimensions, it is also possible to extract from the composite labels and examine the
DATE tagger performance for the individual dimensions. Here we focus on the SPEECH-ACT dimension since, as mentioned above, it is more likely to generalize to $\mathrm{HH}$ travel dialogues and to other task domains. Table 2 shows the results for a DATE tagger trained and tested on only the SPEECH-ACT dimension. The reported accuracies are signifcantly better than the baseline in each case and the differences between the rows are also statistically significant. The results support our original hypothesis, showing that the June-2000 SPEECH-ACT DATE tagger generalizes more readily to the October-2001 corpus, with an accuracy of $82.57 \%$ (Row 2). Furthermore, as before, even a small amount of training data from the 2001 corpus makes a significant improvement in accuracy to $95.68 \%$ (Row 3), which is close to the $99.1 \%$ accuracy (Row 1) reported for training and testing on the June-2000 corpus as estimated by 4fold cross-validation.

Human-Human Results: In order to examine whether there is any generalization from labelled $\mathrm{HC}$ data to $\mathrm{HH}$ data for the same task, we apply a DATE tagger trained on only the SPEECH-ACT dimension. The first row of Table 3 shows that when a DATE tagger is trained on only the HC corpus and tested on the HH corpus that the accuracy is $36.72 \%$ (a significant improvement over the baseline). This result demonstrates quantitatively that the $\mathrm{HC}$ data can be used to improve performance of a tagger for $\mathrm{HH}$ data.

Now, let us consider a situation where we only have $305 \mathrm{HH}$ labelled utterances from 10 of the $\mathrm{HH}$ dialogues to train a DATE tagger. Row 2 shows that we achieve $48.75 \%$ accuracy when testing on the remainder of the $\mathrm{HH}$ corpus. However if we add the $\mathrm{HC}$ data to the training set, the accuracy improves significantly to $55.48 \%$ (Row 3 ). Again this result demonstrates quantitatively that the $\mathrm{HC}$ data can improve performance of a tagger for $\mathrm{HH}$ data.

Row 4 shows that the utility of the $\mathrm{HC}$ corpus decreases if larger amounts of $\mathrm{HH}$ labelled data are available; using $95 \%$ of the data to train and test- 


\begin{tabular}{|l|l|l|ll|}
\hline Training Data & Test Data & Maj. Cl. Baseline & Acc. & (SE) \\
\hline \hline $\begin{array}{l}\text { JUNE-2000 \& OCTOBER- } \\
2001\end{array}$ & CMU-CORPUS & $28.07 \%$ & $36.72 \%$ & $2.76 \%$ \\
\hline 305 CMU-CORPUS & CMU-CORPUS - 305 & $43.93 \%$ & $48.75 \%$ & $1.82 \%$ \\
\hline $\begin{array}{l}\text { JUNE-2000, OCTOBER- } \\
2001 \& 305 \text { CMU-CORPUS }\end{array}$ & CMU-CORPUS - 305 & $28.04 \%$ & $55.48 \%$ & $1.81 \%$ \\
\hline CMU-CORPUS & 20fold Xval CMU-CORPUS & $54.14 \%$ & $76.56 \%$ & \\
\hline
\end{tabular}

Table 3: Results for Identifying DATE Speech-Act Tags in the CMU Human-Human Corpus (Maj. Cl. = Majority Class, Acc. = Accuracy, SE = Standard Error)

ing on $5 \%$ with 20 -fold cross-validation achieves an accuracy of $76.56 \%$.

Examination of the errors that the tagger makes indicates both similarities and differences between $\mathrm{HH}$ and $\mathrm{HC}$ dialogues. For example, information is presented in small installments in the $\mathrm{HH}$ dialogues whereas information presentation utterances in the $\mathrm{HC}$ dialogues tend to be very long. The information presentation utterances in $\mathrm{HH}$ dialogues then appear to be syntactically similar to the implicit confirmations in the HC data. Finally, some utterance types that are very frequent in the $\mathrm{HC}$ data such as instructions rarely occur in the $\mathrm{HH}$ dialogues.

The rules that are learned for a DATE tagger trained on the HC corpora and the HH CMU-corpus for the offer SPEECH-ACT are in Figures 9 and 10. There are two main conclusions that can be drawn from these figures about the generalization from $\mathrm{HC}$ to HH corpora in the SPEECH-ACT dimension. First, in general, a larger number of rules are learned for the $\mathrm{HH}$ data, suggesting that there is greater variation for the same speech act in $\mathrm{HH}$ dialogues. While this is not surprising, there is also significant overlap in the features and values used in the rules. For example, the utterance string feature utilizes words such as select, flight, do, okay, fine, these in both rule sets.

\section{Discussion and Future Work}

In summary our results show that: (1) It is possible to assign DATE dialogue act tags to system utterances in $\mathrm{HC}$ dialogues from many different systems for the same domain with high accuracy; (2) A DATE tagger trained on data from an earlier version of the system only achieves moderate accuracy on a later version of the system without a small amount labelled training data from that later version; (3) Labelled training data from $\mathrm{HC}$ dialogues can improve the performance of a DATE tagger for HH dialogue when only a small amount of $\mathrm{HH}$ training data is available.
Previous work has also reported results for dialogue act taggers, using similar features to those we use, with accuracies ranging from $62 \%$ to $75 \%$ (Reithinger and Klesen, 1997; Shriberg et al., 2000; Samuel et al., 1998). Our best accuracy for the HC data is $98 \%$. The best performance for the HH corpus is $76 \%$ accuracy for the cross-validation study using only $\mathrm{HH}$ data. However, accuracies reported for previous work are not directly comparable to ours for several reasons. First, some of our results concern labelling the system side of utterances in $\mathrm{HC}$ dialogues for the purpose of automatic evaluation of system performance. It is much easier to develop a high accuracy tagger for $\mathrm{HC}$ dialogue than it is for $\mathrm{HH}$ dialogue.

We also applied the DATE tagger to HH dialogue, and focused on the travel agent side of the dialogue. Here the accuracies that we report are more comparable with that of other researchers, but large differences should nevertheless be expected due to differences in the types of corpora, dialogue act tagging schemes, and features used.

We considered the possibility of generating dialogue acts automatically in the logfiles. This idea was attractive because it is possible to easily implement the generation of dialogue acts tags in the logfiles. Large amounts of human-computer data would then be available for the human-human labelling task or for evaluation efforts. However, this turned out to be impractical because we found it difficult to get dialogue designers across the different participating sites to agree on a labelling standard. We therefore believe that machine learning methods for classification such as the one discussed here might still be necessary to automate the tagging task for rapid evaluation and labelling efforts.

As part of the ISLE NSF/EU project, the labelled corpus that we developed for this work will soon be released by the LDC, and other researchers will then be able to utilize it to improve upon our results. In addition, we believe this corpus could be useful as a 
training resource for spoken response generation in dialogue systems. For example, the dialogue act representation can be used to provide a broad range of text-planning inputs for a stochastic sentence planner in the travel domain (Walker et al., 2001b), or to represent the systems' dialogue strategies for reinforcement learning (Walker, 2000; Scheffler and Young, 2002). In future work, we hope to demonstrate that features derived from the labelling of the system side of the dialogue can also improve performance of a dialogue act tagger for the human utterances in the dialogue, and to conduct additional analyses demonstrating the utility of this representation for cross-site evaluation.

\section{Acknowledgments}

Thanks to John Aberdeen and Christy Doran for their contribution of a labelled set of $10 \mathrm{HH}$ dialogues from the CMU-corpus. The work reported in this paper was partially funded by DARPA contract MDA972-99-3-0003.

\section{References}

J. Allen and M. Core. 1997. Draft of DAMSL: Dialog act markup in several layers. Coding scheme developed by the MultiParty group, 1st Discourse Tagging Workshop, Univ. of Penn, March 1996.

R. Cattoni, M. Danieli, A. Panizza, V. Sandrini, and C. Soria. 2001. Building a corpus of annotated dialogues: the ADAM experience. In Proc. of the Conference Corpus-Linguistics-2001, Lancaster, U.K.

W. Cohen. 1996. Learning trees and rules with setvalued features. In 14th Conference of AAAI.

B. Di Eugenio, P. W. Jordan, J. D. Moore, and R. H. Thomason. 1998. An empirical investigation of collaborative dialogues. In ACL-COLING98, Proc. of the 36th ACL Conference.

C. Doran, J. Aberdeen, L. Damianos, and L. Hirschman. 2001. Comparing several aspects of human-computer and human-human dialogues. In SIGDIAL Workshop in conjuction with Eurospeech 2001.

M. Eskenazi, A. Rudnicky, K. Gregory, P. Constantinides, R. Brennan, K. Bennett, and J. Allen. 1999. Data collection and processing in the carnegie mellon communicator. In Proc. of Eurospeech-99, pages 2695-2698.

M. Finke, M. Lapata, A. Lavie, L. Levin, L. Mayfield Tomokiyo, T. Polzin, K. Ries, A. Waibel, and K. Zechner. 1998. Clarity: Inferring discourse structure from speech. In AAAI Symposium on Applying Machine
Learning to Discourse Processing Proceedings, Stanford, California.

H. Hastie, R. Prasad, and M. A. Walker. 2002. Automatic evaluation: Using a date dialogue act tagger for user satisfaction and task completion prediction. In LREC 2002.

A. Isard and J. C. Carletta. 1995. Replicability of transaction and action coding in the map task corpus. In M. A. Walker and J. Moore, eds., AAAI Spring Symposium: Empirical Methods in Discourse Interpretation and Generation, pages 60-67.

P. W. Jordan. 2000. Intentional Influences on Object Redescriptions in Dialogue: Evidence from an Empirical Study. Ph.D. thesis, Intelligent Systems Program, University of Pittsburgh.

N. Reithinger and M. Klesen. 1997. Dialogue act classification using language models. In Proc. of $\mathrm{Eu}$ rospeech '97, pages 2235-2238, Rhodes, Greece.

K. Samuel, S. Carberry, and K. Vijay-Shanker. 1998. Dialogue act tagging with transformation-based learning. In Proc. of COLING-ACL, pages 1150-1156.

K. Scheffler and S. Young. 2002. Automatic learning of dialogue strategy using dialogue simulation and reinforcement learning. In HLT Conference.

E. Shriberg, P. Taylor, R. Bates, A. Stolcke, K. Ries, D. Jurafsky, N. Coccaro, R. Martin, M. Meteer, and C. Van Ess-Dykema. 2000. Can prosody aid the automatic classification of dialog acts in conversational speech. Language and Speech: Special Issue on Prosody and Conversation.

M. A. Walker, D. Litman, C. A. Kamm, and A. Abella. 1997. PARADISE: A general framework for evaluating spoken dialogue agents. In Proc. of ACL/EACL 97, pages 271-280.

M. A. Walker, J. Aberdeen, J. Boland, E. Bratt, J. Garofolo, L. Hirschman, A. Le, S. Lee, S. Narayanan, K. Papineni, B. Pellom, J. Polifroni, A. Potamianos, P. Prabhu, A. Rudnicky, G. Sanders, S. Seneff, D. Stallard, and S. Whittaker. 2001a. Darpa communicator dialog travel planning systems: The june 2000 data collection. In Proc. of EUROSPEECH 2001.

M. A. Walker, O. Rambow, and M. Rogati. 2001b. Spot: A trainable sentence planner. In Proc. of NAACL.

M. A. Walker, R. Passonneau, and J. E. Boland. 2001c. Quantitative and qualitative evaluation of DARPA communicator spoken dialogue systems. In Proc. of ACL 2001.

M. A. Walker. 2000. An application of reinforcement learning to dialogue strategy selection in a spoken dialogue system for email. Journal of Artificial Intelligence Research, 12:387-416. 


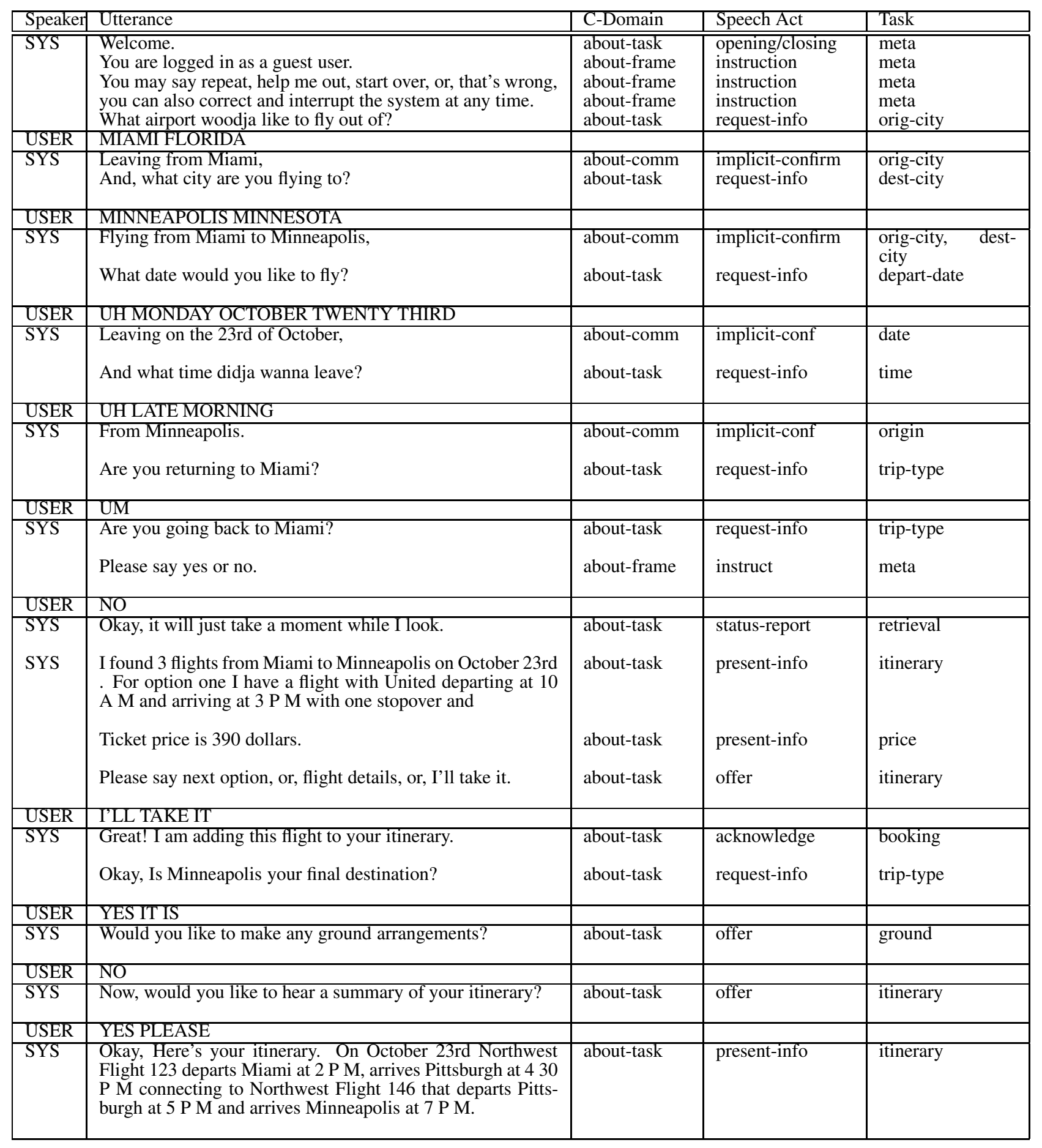

Figure 5: Dialogue Illustrating the Conversational-Domain, Speech-Act, and Task-Subtask Dimensions of DATE 
if left-context $1=S P A-p r e s e n t-i n f o ~ \wedge$ pattern-length $\leq 25 \wedge$ position-in-turn $\geq 2 \wedge$ right-sys-utt-string contains none

or if left-context $2=$ SPA-present-info-SPA-present-info $\wedge$ system-name=ATT

or if right-sys-utt-string contains also $\wedge$ left-sys-utt-string contains at

or if utt-string contains select

or if utt-string contains confirm

or if utt-string contains fine

or if right-sys-utt-string contains locations $\wedge$ utt-string contains If

or if left-context2=SPA-implicit-confirm-SPA-instruction $\wedge$ utt-string contains Which

or if utt-string contains Okay $\wedge$ utt-string contains flight

or if left-context $2=$ SPA-explicit-confirm-SPA-acknowledgement $\wedge$ utt-string contains flight

or if utt-string contains these $\wedge$ utt-string contains Are

or if rec-orig-string contains sixteenth $\wedge$ utt-string contains Do

then offer

Figure 9: Rules learned for DATE SPEECH-ACT offer using June-2000 plus 2000 Examples of October-2001 as Training

if left-sys-utt-string contains 'NUMBER' $\wedge$ pattern-length $\leq 25 \wedge$ right-sys-utt-string contains none $\wedge$ utt-string contains OK or if position-in-turn $\geq 2 \wedge$ left-sys-utt-string contains dollars $\wedge$ pattern-length $\leq 55 \wedge$ contains-word-CITY-or-AIRPORT=false or if utt-string contains this $\wedge$ pattern-length $\leq 37 \wedge$ contains-word-FLIGHT-or-AIRLINE=true

or if left-sys-utt-string contains per $\wedge$ da-num $\leq 2$

or if right-sys-utt-string contains rate

or if utt-string contains these

or if utt-string contains itinerary $\wedge$ pattern-length $\leq 41$

or if utt-string contains reservation

or if utt-string contains select

or if utt-string contains book $\wedge$ utt-string contains it

or if utt-string contains whether

or if utt-string contains $\mathrm{OK} \wedge$ utt-string contains Is

or if utt-string contains MAKE

or if utt-string contains one $\wedge$ right-sys-utt-string contains none

or if utt-string contains fine

or if utt-string contains Kay

or if right-sys-utt-string contains locations

or if utt-string contains THE $\wedge$ utt-string contains LIKE

or if left-sys-utt-string contains over $\wedge$ utt-string contains flight $\wedge$ utt-string contains would

or if utt-string contains take $\wedge$ utt-string contains Do

or if left-sys-utt-string contains yes $\wedge$ utt-string contains what $\wedge$ utt-string contains flight

then offer

Figure 10: Rules learned for DATE SPEECH-ACT offer using 305 CMU-Corpus Utterances as Training 